\title{
SIMULADORES COMPUTADORIZADOS E AUTO-PERCEPÇÃO DE CONDUTA DE ALUNOS E ALUNAS DE PSICOLOGIA*
}

\author{
COMPUTERIZED SIMULATORS \\ AND SELF-PERCEIVED CONDUCT OF \\ MALE AND FEMALE STUDENTS OFPSYCHOLOGY
}

Ebenézer A. de OLIVEIRA**

Universidade Federal de Santa Maria

\begin{abstract}
RESUMO
Este estudo investigou os efeitos da introdução de atividades laboratoriais com um simulador computadorizado sobre a Freqüência Escolar e as auto-percepções dos estudantes de graduação em Psicologia quanto ao Interesse, Compreensão do conteúdo curricular e Cooperação com pares. Os efeitos do Sexo dos estudantes também foram examinados, sendo a amostra constituída por 18 alunas e 5 alunos. Conforme previsto, todas as variáveis apresentaram um aumento médio em função do uso do simulador. Houve ainda uma interação significativa entre Sexo e Simulador sobre a Freqüência Escolar, indicando que os alunos se beneficiaram do novo recurso pedagógico mais do que as alunas. As alunas, em contrapartida, apresentaram maiores índices de Freqüência e de Cooperação do que os alunos, independentemente do currículo. Esses resultados são discutidos em termos de ensino-aprendizagem do terceiro grau e de estereótipos de papéis sexuais.
\end{abstract}

Palavras-chave: ensino de terceiro grau; informatização da educação; papéis sexuais

ABSTRACT

This study tested the effects of introducing lab activities with a computerized simulator on undergraduate Psychology students' School Attendance and

(*) Pesquisa financiada pelo FIPE - Fundo de Incentivo à Pesquisa, da Universidade Federal de Santa Maria. O autor agradece aos alunos do curso de Psicologia da UFSM que participaram voluntariamente deste estudo e à monitora Giana B. Frizzo, que coletou os dados.

${ }^{* *}$ Endereço para correspondência: Departamento de Psicologia da Universidade Federal de Santa Maria - Rua Floriano Peixoto, 1750 - Sala 309. 97015-372 Santa Maria, RS. E-mail: ebe@ccsh.ufsm.br 
SIMULADORES COMPUTADORIZADOS E AUTO-PERCEPÇÃO DE...

self-perceived Interest, Comprehension of curriculum materials, and Peer Cooperation. Sex effects were also examined in the 18 female and 5 male students that participated. As predicted, all variables presented a mean increase as a function of simulator use. There was also a significant interaction between Sex and Simulator on Attendance, suggesting that male students benefited better than their female counterparts from the new pedagogical tool. The female students, on the other hand, showed higher indices of Attendance and Cooperation than the male students, regardless of curriculum materials. These results are discussed in terms of undergraduate teaching/learning and sex-role stereotypes.

key words: undergraduate teaching; informatization of education; sex roles

Desde que Skinner lançou a famosa máquina de ensino nos Estados Unidos, o rápido desenvolvimento tecnológico vem inspirando novas estratégias pedagógicas que utilizam microcomputadores não apenas para ensinar fatos básicos de disciplinas acadêmicas, mas também para facilitar a aquisição, por parte do aprendiz, das chamadas cognições de "alta ordem" (e.g., compreensão e aplicação de conceitos complexos, estratégias de solução de problemas variados, criação de textos, etc.). Dentre as novas estratégias de ensino-aprendizagem pela informática, destacam-se os programas tutoriais, jogos, utilitários, solucionadores de problemas e simuladores (Slavin, 1994; Valente, 1991).

Os programas tutoriais põem 0 microcomputador como substituto do professor, tendo a vantagem da individualização do ritmo e do nível de diálogo mantido entre o estudante e a máquina. Os jogos educacionais despertam o interesse do estudante com temas de aventura e situações arriscadas, e geralmente envolvem objetivos específicos a serem atingidos segundo determinadas regras que 0 estudante deve seguir inteligentemente. Já os programas utilitários empregam o computador como um instrumento útil para a execução de tarefas tais como composição e edição de textos e representação tabular e gráfica de dados.

Os programas voltados especificamente para soluções de problemas tipicamente perm item que o aprendiz, e não o professor ou 0 computador, escolha a situação-problema a ser resolvida. Ao programar o computador segundo uma linguagem convencional (e.g., BASIC, Logo, Pascal), o estudante define a situação-problema, em algum domínio que the interessa, o objetivo a ser alcançado e os passos necessários para se chegar a tal objetivo. Finalmente, os simuladores computadorizados provêm modelos de alguma parte do "mundo real," em algum estado ou em algum momento específico. A vantagem deste recurso pedagógico é a produção de efeitos realísticos de situações que seriam muito arriscadas, onerosas ou, por diversas razões, impraticáveis no mundo real, como ocorre em alguns experimentos científicos. Trabalhando geralmente em pequenos grupos, os estudantes propõem hipóteses, testam-nas, analisam e interpretam os resultados, e, a partir destes, abandonam, modificam ou confirmam o seu modo de pensar sobre o universo.

Nos Estados Unidos da América, mais de 97\% das escolas públicas já adotam esses recursos da informática na prática de ensino-aprendizagem (U.S. Bureau of the Census, 1994), enquanto que no Brasil tais recursos continuam sendo mais um privilégio das escolas particulares (Aguiar, 1989). Desde 1981, contudo, uma série de iniciativas do Ministério da Educação (MEC), da Secretaria Especial de Informática (SEI), do Conselho Nacional de Desenvolvimento Científico e Tecnológico (CNPq) e, mais tarde, da Comissão Especial de Informática na Educação (CEI), permitiu a im- 
plantação de alguns centros de pesquisa em universidades públicas brasileiras, abrindo as portas da informatização às camadas sociais menos favorecidas. Nota-se, na vasta literatura emergente dessa pesquisa, grande ênfase na eficácia pedagógica do ensino-aprendizado de primeiro e segundo graus, com a linguagem Logo (Aguiar, 1989; Valente, 1991).

Em contraste, a informatização do ensino de terceiro grau, particularmente, do curso de Psicologia, ainda atravessa uma fase de implantação, haja vista os indícios de indiferença ou oposição contra as novas tecnologias, por parte de docentes e profissionais dessa área (Andriola, 1996; Peres, 1997). Ainda não se sabe, por exemplo, se o uso de simuladores computadorizados no ensino-aprendizado da Psicologia refletiria no aumento da freqüência escolar. E, embora se reconheça que os simuladores têm potenciais "muito mais ambiciosos do que os demais CAis [modelos de instrução auxiliada pelo computador]" (Valente, 1991, p. 20), ainda não se tem explorado empiricamente o papel de tais recursos tecnológicos na percepção do estudante sobre sua própria conduta.

Segundo a teoria cognitiva social de Albert Bandura (1986; 1997), em condições desafiantes tais como as do ensino-aprendizado, as percepções que o estudante desenvolve sobre os seus próprios processos mentais e sobre a situação que se lhe apresenta podem sertão importantes quanto a sua capacidade objetiva para obter o sucesso. Em particular, os níveis auto-avaliativos de interesse e de compreensão dos requisitos de uma tarefa podem ser decisivos na manutenção do engajamento do indivíduo e, por conseguinte, do seu êxito. E, conforme sugere Slavin (1991), a disposição de cooperar com colegas implica tanto na melhora do desempenho em tarefas desafiantes, quanto na superior qualidade de relacionamento interpessoal, embora lamentavelmente ainda poucos professores façam uso de atividades cooperativas em sala de aula. Resta-nos saber se o simulador computadorizado funciona como um catalisador da melhora de interesse acadêmico e dos níveis de compreensão e de cooperação com pares, segundo percebem os estudantes de Psicologia.

Uma outra questão pouco explorada na literatura diz respeito aos possíveis efeitos diferenciais do uso de simulador computadorizado entre os sexos. Embora a falta de respaldo teórico e empírico impeça uma formulação clara de hipóteses a priori neste estudo, há pelo menos duas razões para se suspeitar que alunos e alunas reagem diferentemente à introdução do simulador computadorizado no ensinoaprendizado de Psicologia.

Em primeiro lugar, há evidência que, desde as séries do ensino fundamental, os meninos recebem mais reforço positivo por seu desempenho intelectual do que as meninas, o que deixa os meninos menos vulneráveis do que as meninas ao desamparo aprendido (Dweck, Davidson, Nelson, \& Enna, 1978), especialmente em situações novas em que há dúvidas sobre a obtenção de um desempenho satisfatório. Em segundo lugar, conforme indica Lepper (1985), nota-se um favoritismo pelo sexo masculino na oportunização de carreiras que empregam alta tecnologia, o que resulta numa maior representação de homens do que de mulheres em profissões que empregam diretamente o computador. Em conjunto, tais evidências levam a crer que a novidade tecnológica do simulador pode atrair e beneficiar mais o estudante de Psicologia do sexo masculino do que do sexo feminino.

O presente estudo parte da pressuposição de que atividades laboratoriais que simulam o condicionamento comportamental podem facilitar a compreensão de conceitos introduzidos em sala de aula, além de aumentar os níveis de interesse e de participação dos estudantes de Psicologia. Embora intuitivamente lógica, tal suposição até hoje se apóia em depoimentos informais, partindo dos próprios criadores de softwares (e.g., Clay, 1997). Mais especificamente, este estudo testou experimentalmente a hipótese de que a introdução do uso de um simulador computadorizado, em ativida- 
SIMULADORES COMPUTADORIZADOS E AUTO-PERCEPÇÃO DE...

des laboratoriais complementares ao ensino regular, aumenta o percentual de freqüência escolar e a auto-percepção da compreensão de conceitos teóricos e dos níveis de interesse e de cooperação entre os estudantes de psicologia.

\section{MÉTODO}

Empregou-se um delineamento experimental de medidas repetidas intraparticipantes, com a diferença intraparticipante determinada pelo não uso vs. uso de um simulador computadorizado, e a diferença entre blocos de sujeitos determinada pelo sexo dos participantes.

\section{Participantes}

Participaram voluntariamente deste estudo 23 alunos (92\%) matriculados no curso de Psicologia Experimental I, no primeiro semestre letivo de 1998, na Universidade Federal de Santa Maria. Destes, 18 eram do sexo feminino e 5 do sexo masculino; a média de idade era 19,7 anos e a média de horas-aula semanais era 31,56 .

\section{Procedimento e Medidas}

Além de informarem sua idade, sexo e carga horária semanal, os participantes atribuíram a si mesmos notas de 0 (ausência do atributo) a 100 (nível máximo do atributo) quanto ao Interesse, Compreensão e Cooperação com colegas, em três tempos de medição, dos quais apenas os dois primeiros foram utilizados neste estudo. A partir do registro de presença, foi calculado o percentual de Freqüência escolar dos alunos na disciplina de Psicologia Experimental I, relativo ao período letivo. As medições dessas variáveis dependentes aconteceram, aproximadamente, decorrido $1 / 3$ do semestre, quando o currículo contava apenas com aulas teóricas, e novamente duas sema- nas após se introduzirem as práticas laboratoriais com o simulador computadorizado, já na segunda metade do semestre letivo. Todos os dados foram coletados pela monitora da disciplina, até então cega para os objetivos do presente estudo, a qual assegurou aos participantes que os dados seriam mantidos em sigilo e não afetariam suas notas escolares.

O simulador computadorizado foi "Sniffy, the Virtual Rat" (Krames, Graham \& Alloway, 1996), que perm ite o controle do comportamento de um ratinho virtual através de exercícios de moldagem ("shaping") e do emprego de diversos esquemas de reforço e extinção.

\section{Resultados}

Uma série de análises de vanancia (ANOVA) duplas foi realizada, cruzando Sexo com Simulador (i.e., tempos de medição antes e após se introduzir o simulador). Por causa de uma inclinação negativa na distribuição dos escores, as análises foram feitas, primeiramente, usando-se os escores transformados exponencialmente, como tentativa de se corrigir a falta de normalidade na distribuição (Glass \& Hopkins, 1984). Os escores não-transformados também foram analisados.

Como os resultados de ambas as análises foram semelhantes, optou-se por relatar os últimos, pois dados estatisticamente transformados são mais difíceis de se interpretar.

A Tabela 1 apresenta as estatísticas descritivas das variáveis dependentes, antes e após a introdução do simulador computadorizado. As Tabelas 2 a 5 mostram os resultados das ANOV As, com um alfa de ,05.

Conforme previsto, os alunos relataram um aumento médio em sua percepção dos níveis de Interesse (ver Tabela 3), Compreensão (ver Tabela 4) e Cooperação com colegas (ver Tabela 5). Digno de nota é o efeito significante da interação Sexo X Simulador sobre a Freqüência, conforme indicado na Tabela 2. 
Tabela 1 - Médias (M) e Desvios Padrão (DP) das Variáveis Dependentes ( $N=23)$

\begin{tabular}{llccc}
\hline & $\begin{array}{c}\text { Tempo de Medição 1 } \\
\text { (sem simulador) }\end{array}$ & DP & $\begin{array}{c}\text { Tempo de Medição 2 } \\
\text { (com simulador) }\end{array}$ \\
\hline \multicolumn{1}{c}{ Variáveis } & M & M & DP \\
\hline 1. Freqüência & 94,04 & 7,11 & 98,48 & 4,63 \\
2. Interesse & 85,17 & 10,26 & 92,70 & 6,80 \\
3. Compreensão & 79,21 & 10,89 & 91,09 & 7,68 \\
4. Cooperação & 83,96 & 12,19 & 90,35 & 10,14 \\
\hline
\end{tabular}

Tabela 2 - ANOVA dos efeitos de Sexo e Uso de Simulador sobre Freqüência $(N=23)$

\begin{tabular}{lcrcc}
\hline Fonte de Variância & Soma dos Quadrados & $\mathbf{9}^{1}$ & Quadrados Médios & F \\
\hline 1. Sexo & 231,98 & 1 & 231,98 & $5,26^{*}$ \\
2. Resíduo & 926,89 & 21 & 44,14 & \\
3. Simulador & 325,02 & 1 & 325,02 & $20,88^{* *}$ \\
4. Sexo X Simulador & 98,94 & 1 & 98,94 & $6,36^{*}$ \\
5. Resíduo & 326,89 & 21 & 15,57 & \\
\hline
\end{tabular}

NOTA. ${ }^{*} \mathbf{P}<, 05 ;{ }^{* \star} \mathbf{P}<, 001$

Tabela 3 - ANOVA dos efeitos de Sexo e Uso de Simulador sobre Interesse $(\mathrm{N}=23)$

\begin{tabular}{lcrcr}
\hline Fonte de Variância & Soma dos Quadrados & $\mathbf{9}^{1}$ & Quadrados Médios & $\mathbf{F}$ \\
\hline 1. Sexo & 197,83 & 1 & 197,83 & 1,88 \\
2. Resíduo & $2.207,47$ & 21 & 105,12 & $15,91^{*}$ \\
3. Simulador & 653,63 & 1 & 653,63 & $1,56^{*}$ \\
4. Sexo X Simulador & 64,06 & 1 & 64,06 & 41,09 \\
5. Resíduo & 862,81 & 21 &
\end{tabular}

NOTA. * $\mathbf{P}<, 05 ;{ }^{\star *} \mathbf{P}<, 001$

Tabela 4 - ANOVA dos efeitos de Sexo e Uso de Simulador sobre Compreensão ( $N=23)$

\begin{tabular}{lcrrr}
\hline Fonte de Variância & Soma dos Quadrados & $\mathbf{9}^{1}$ & Quadrados Médios & $\mathbf{F}$ \\
\hline 1. Sexo & 16,96 & 1 & 16,96 & 0,16 \\
2. Resíduo & $2.266,47$ & 21 & 107,93 & $16,31^{*}$ \\
3. Simulador & $1.250,15$ & 1 & $1.250,15$ & 0,19 \\
4. Sexo X Simulador & 14,50 & 1 & 14,50 & 76,66 \\
5. Resíduo & $1.609,81$ & 21 &
\end{tabular}

NOTA. *P $<, 001$ 
SIMULADORES COMPUTADORIZADOS EAUTO-PERCEPÇÃO DE...

Tabela 5 - ANOVA dos efeitos de Sexo e Uso de Simulador sobre Cooperação $(N=23)$

\begin{tabular}{lcrrr}
\hline Fonte de Variância & Soma dos Quadrados & gl & Quadrados Médios & F \\
\hline 1. Sexo & $1.070,30$ & 1 & $1.070,30$ & $7,06 "$ \\
2. Resíduo & $3.184,14$ & 21 & 151,63 & \\
3. Simulador & 305,71 & 1 & 305,71 & $5,03 "$ \\
4. Sexo X Simulador & 0,49 & 1 & 0,49 & 0,01 \\
5. Resíduo & $1.277,25$ & 21 & 60,82 & \\
\hline
\end{tabular}

NOTA $.{ }^{*} \mathbf{P}<, 05 ;{ }^{* *} \mathbf{P}=, 01$

Observa-se na Tabela 2 que as alunas apresentaram, em média, maior Freqüência às aulas do que os alunos, independentemente do uso de atividades laboratoriais com o simulador computadorizado. Igualmente, houve um efeito principal de Sexo sobre o nível de Cooperação, favorecendo o sexo feminino (ver Tabela 5).

\section{Discussão}

Os resultados confirmam a hipótese inicial de que exercícios laboratoriais com simulador computadorizado aumentam a freqüência escolar dos estudantes de Psicologia e sua autopercepção sobre os níveis de Interesse, Compreensão e Cooperação. Embora a freqüência escolar dos alunos tendeu a aumentar mais do que a das alunas, em função do uso de simulador, os resultados se generalizaram entre os sexos, corroborando o que já se conhece sobre a eficácia pedagógica de simuladores eletrônicos no ensino-aprendizado de primeiro e segundo graus.

Vale salientar que o simulador usado neste estudo foi um complemento, e não um substituto das atividades didáticas de sala de aula. Como afirma Slavin (1994), esse recurso tecnológico "é freqüentemente mais eficaz quando usado em acréscimo à instrução regular de sala de aula, mas tem efeitos mais brandos e menos consistentes se utilizado no lugar da instrução de sala de aula" (p. 339; ver também Valente, 1991). Outrossim, a verossimilhança do simulador empregado neste estudo não é de se desprezar (Clay, 1997; Krames, Graham, \& Alloway, 1996), e bem pode ter contribuído para os favoráveis resultados.

Desnecessário dizer que o aumento da freqüência escolar e da percepção dos alunos quanto aos seus níveis de Interesse, Compreensão e Cooperação com pares é de fundamental importância pedagógica. Alunos mais assíduos e interessados no processo de aprendizagem, e que se julgam bons entendedores e cooperadores com seus pares provavelmente terão menor chance de fracassar ou de abandonar a universidade. O presente estudo, portanto, deixa-nos ainda mais perplexos com a resistência de muitos docentes das ciências sociais e humanas quanto à utilização de ferramentas tecnológicas pedagogicamente importantes. Ao examinar esta questão numa universidade pública, Peres (1997) conclui que a falta de familiaridade com os mais modernos recursos tecnológicos e o comodismo associado ao emprego estável e seguro podem estar influenciando. Em contrapartida, essa pesquisadora encoraja projetos interdisciplinares que permitam maior intercâmbio dos docentes das diversas áreas, a fim de que novos recursos tecnológicos estejam cada vez mais ao alcance de todos.

Os resultados do presente estudo também indicam que as alunas são mais assíduas e se julgam mais cooperativas com seus pares do que os alunos, independentemente do uso ou não de atividades com simulador computa- 
dorizado. Isso parece surpreendente, considerando-se o esterótipo social e alguns indicadores empíricos de que os homens geralmente se envolvem mais do que as mulheres em atividades científicas, especialmente quando se emprega o computador (ver Lepper, 1985). Por outro lado, os resultados também sugerem que a assiduidade relativa dos alunos se beneficia mais do uso de simulador computadorizado que a das alunas; ou seja, os alunos tendem a aumentar consideravelmente mais sua freqüência às aulas do que as alunas, em função do acréscimo do simulador ao currículo.

Duas explicações para esse quadro são possíveis, sendo uma teórica e outra metodológica. Primeiramente, é possível que as alunas em questão simplesmente estejam cumprindo o papel pró-social que lhes é socialmente atribuído. Já que tradicionalmente as alunas são socializadas para "mitigar conflitos ..., promover concordância e restaurar ou manter o bom funcionamento do grupo" (Maccoby, 1990, p. 516), sua maior freqüência às aulas e cooperatividade auto-percebida não são surpreendentes. Por outro lado, os resultados também indicam que os alunos, mais do que as alunas, estão regulando o seu comparecimento às aulas em função do que se lhes é oferecido em termos de atividades curriculares, e isso bem pode estar refletindo a auto-defesa de interesses pessoais, típica do sexo masculino (Maccoby, 1990).

Do ponto de vista metodológico, os resultados referentes à freqüência escolar podem ser explicados pelo efeito de teto ("ceiling effect") sobre o percentual de freqüência das alunas, que já era bastante elevado no primeiro tempo de medição (96\%), faltando, portanto, uma margem para variação.

Essa discussão aponta para certas limitações do presente estudo, em que se baseiam recomendações para futuras investigações. Primeiro, a chance de um efeito de teto (especialmente sobre a freqüência relativa das alunas) seria menor se os dados fossem coletados num espaço de tempo maior. Uma outra limitação deste estudo é o emprego de uma ordem fixa e limitada dos níveis experimentais, que permitiu avaliar os efeitos imediatos do acréscimo do simulador computadorizado ao currículo, mas deixou sem respostas outras questões igualmente importantes, tais como a duração desses mesmos efeitos e o que aconteceria às variáveis dependentes uma vez retirado o simulador. Tais questões aguardam novas investigações, preferencialmente com reversão experimental.

\section{CONCLUSÃO}

Em suma, o presente estudo permite-nos chegar às seguintes conclusões com respeito aos efeitos de simuladores computadorizados e do sexo dos estudantes de Psicologia sobre suas condutas auto-percebidas em sala de aula:

(1) Independentemente de sexo, os estudantes tendem a aumentar sua auto-percepção dos níveis de interesse, compreensão e cooperação.

(2) Embora alunos de ambos os sexos tendam a melhorar sua freqüência escolar, esta tendência é mais acentuada no sexo masculino do que no sexo feminino.

(3) As alunas tendem a freqüentar mais as aulas e a se julgar mais cooperativas com pares do que os alunos, quer sejam utilizados simuladores computadorizados ou não.

\section{REFERÊNCIAS}

AGUIAR, P. (1989). O uso do computador por crianças com dificuldades de aprendizado. Monografia de especialização. Faculdade de Filosofia, Ciências e Letras Imaculada Conceição. Santa Maria, RS.

ANDRIOLA, W. B. (1996). O uso de computadores na avaliação psicológica: Verificação de sua influência sobre o desempenho individual num teste de raciocínio verbal (RV).

Psicologia: Reflexão e Crítica, 9, 257-268. 
BANDURA, A. (1986). Social foundations of thought and action: A social cognitive theory. Englewood Cliffs, NJ: Prentice-Hall.

BANDURA, A. (1997). Self-efficacy: The exercise of control. New York: Freeman.

CLAY, R. A. (1997). A new era of teaching tools engages students. The APA Monitor, August, 1-3.

DWECK, C. S., Davidson, W., Nelson, S. \& Enna, B. (1978). Sex differences in learned helplessness: I. Differential debilitation with peer and adult evaluators. Developmental Psychology, 14, 268-276.

GLASS, G. V. \& Hopkins, K. D. (1984).Statistical methods in education and psychology. $2^{\text {nd }}$.ed. Englewood Cliffs, NJ: Prentice-Hall.

KRAMES, L., Graham, J. \& Alloway, T. (1996). Sniffy the virtual rat. Version 4.5 for Windows. Pacific Grove, CA: Brooks/Cole Publishing Co.

LEPPER, M. R. (1985). Microcomputers in education: Motivational and social issues. American Psychologist, 40,1-18.
MACCOBY, E. E. (1990). Gender and relationships: A developmental account. American Psychologist, 45, 513-520.

PERES, R. U. (1997). Novas tecnologias da informação: Atitudes de docentes de ciências sociais e humanas. Dissertação de mestrado. Pontífica Universidade Católica de Campinas, Campinas.

SLAVIN, R. E. (1991). Synthesis of research on cooperative learning. Educational Leadership, February, 71-82.

SLAVIN, R. E. (1994). Educational psychology: Theory and practice. Fourth ed. Needham Heights, MA: Allyn \& Bacon.

U.S. Bureau of the Census (1994). Statistical abstracts of the United States. 114th ed. Washington, DC: U.S. Government Printing Office.

VALENTE, J. A. (1991). Usos do computador na educação. Em J. A. Valente (Org.), Liberando a mente: Computadores na educação especial. Campinas: UNICAMP. 